ANÁLISIS [ ISSN: 0120-8454 [ Vol. 49 / No. 90 [ Bogotá, ene.-jun. / 2017 [ pp. 17-37

\title{
La performance del sistema educativo argentino en las últimas décadas*
}

\author{
Analía Otero** \\ Agustina Córica***
}

Recibido: 20 de agosto de 2016 - Aprobado: 25 de octubre de 2016

\section{Resumen}

En el ámbito de expansión y múltiples transformaciones del sistema educativo argentino, sobrevuelan las discusiones sobre la ampliación institucional, la evolución de la población escolarizada y las muestras de un sistema tendente a generar mayor inclusión.

El objetivo de este estudio fue analizar las especificidades de la situación educativa de la población de jóvenes entre 15 y 29 años de edad, en las últimas décadas. En función de ello, se analizó la información disponible acerca de la asistencia y composición del sistema educativo, tanto del nivel medio como del nivel superior. Los hallazgos ratifican la tendencia a una mayor participación de los jóvenes en el sistema educativo, junto a un incremento en el acceso a niveles educativos superiores en la primera década del siglo XXI. En este contexto, el nivel socioeconómico persiste como un factor de peso en las trayectorias educativas diferenciales, con saldos pendientes en materia de inclusión educativa.

Palabras clave: educación media, educación superior, sistema educativo, trayectorias, transiciones.

* $\quad$ Artículo de investigación. El trabajo está adscrito en el Programa de Investigaciones en Juventud, de la Facultad Latinoamericana de Ciencias Sociales (Flacso, sede Argentina), y fue realizado en el marco del Programa de Internalización de la Educación Superior y Cooperación Internacional, y con financiamiento de la convocatoria Núcleo de Estudios e Investigaciones en Educación Superior del Sector Educativo del Mercosur. DOI: http://dx.doi.org/10.15332/ s0120-8454.2017.0090.01

* Doctora en Ciencias Sociales. Investigadora adjunta del Consejo Nacional de Investigaciones Científicas y Técnicas. Investigadora principal del Programa de Investigaciones en Juventud de la Facultad Latinoamericana de Ciencias Sociales. Código postal: Tucumán 1966, C1050AAN, Ciudad Autónoma de Buenos Aires. Correo electrónico: aotero@ flacso.org.ar

** Doctora en Ciencias Sociales. Investigadora arincipal del Programa de Investigaciones en Juventud de la Facultad Latinoamericana de Ciencias Sociales y becaria posdoctoral del Consejo Nacional de Investigaciones Científicas y Técnicas. Código postal: Olivieri 212 PA,Ciudad Autónoma de Buenos Aires. Correo electrónico: acorica@flacso.org.ar 


\title{
The performance of Argentine education system in recent decades*
}

\author{
Analía Otero** \\ Agustina Córica***
}

\section{A bstract}

In the context of expansion and multiple transformations of the Argentine educational system, overfly discussions on institutional expansion, the evolution of the school population and samples of a system designed to generate greater inclusion. The aim of the research was to analyze the specificities of the educational status of the population of 15 to 29 year-old in recent decades. On that basis, it analyzes information available about assistance and composition of the educational system considering both the middle level and the upper level. The findings confirm the trend of an increased participation of young people in the education system together with an increase in the access to higher education levels in the first decade of the century. In this context, the socio-economic level persists as a factor in the differential educational trajectories, together with outstanding balances on educational inclusion.

Keywords: Secondary education, higher education, education system, paths, transitions.

Research article. The work is attached to the Youth Research Program of the Latin American Faculty of Social Sciences (Flacso, Argentina), and was carried out within the framework of the Internalization Program of Higher Education and International Cooperation, and with funding from the invitation Center for Studies and Research in Higher Education of the Educational Sector of Mercosur. DOI: http://dx.doi.org/10.15332/s0120-8454.2017.0090.01

* PhD in Social Sciences, Deputy Researcher of the National Council of Scientific and Technical Research. Principal Investigator of the Youth Research Program of the Latin American Faculty of Social Sciences. Postal code: Tucumán 1966, C1050AAN, Ciudad Autónoma de Buenos Aires. Email: aotero@flacso.org.ar

* PhD in Social Sciences, Principal Investigator of the Youth Research Program of the Latin American Faculty of Social Sciences and Postdoctoral Fellow of the National Council of Scientific and Technical Research. Postal code: Olivieri 212 PA Ciudad Autónoma de Buenos Aires. Email: acorica@flacso.org.ar 


\title{
La performance du système éducatif argentin au cours des dernières décennies*
}

\author{
Analía Otero** \\ Agustina Córica ${ }^{* * *}$
}

\section{Résumé}

Dans le domaine de l'élargissement et des multiples transformations du système éducatif argentin, surviennent les discussions sur l'extension institutionnelle, l'évolution de la population scolarisée et les échantillons d'un système qui tend à générer une plus grande inclusion.

L'objectif de cette étude a été d'analyser les spécificités de la situation éducative de la population de jeunes entre 15 et 29 ans, au cours des dernières décennies. En fonction de cela, l'information disponible au sujet de l'assistance et de la composition du système éducatif, tant au niveau secondaire comme supérieur, fut analysée. Les découvertes confirment la tendance vers une plus grande participation des jeunes dans le système éducatif, accompagnée d'une augmentation de l'accès aux niveaux éducatifs supérieurs dans la première décennie du XXIe siècle. Dans ce contexte, le niveau socio-économique persiste comme un facteur de poids dans les trajectoires éducatives différentielles, avec des dettes en matière d'inclusion éducative.

Mots clés: Éducation secondaire, éducation supérieure, système éducatif, trajectoires, transitions.

Article de recherche. Ce travail est adscrit au Programme de Recherches sur la Jeunesse de la Faculté Latino-Américaine de Sciences Sociales (Flacso, en Argentine) et fut réalisé dans le cadre du Programme d'Internalisation de l'Éducation Supérieure et Coopération Internationale, avec le financement du concours Noyau d'Études et de Recherches sur l'Éducation Supérieure du Secteur Éducatif du Mercosur. DOI: http://dx.doi.org/10.15332/s0120-8454.2017.0090.01 *

* Docteur en Sciences Sociales, Chercheuse Adjointe du Conseil National de Recherches Scientifiques et Techniques. Chercheuse Principale du Programme de Recherches sur la Jeunesse de la Faculté Latino-Américaine de Sciences Sociales. Code postal: Tucumán 1966, C1050AAN, Ville autonome de Buenos Aires. Email: aotero@flacso.org.ar

*** Docteur en Sciences Sociales, Chercheuse Principale du Programme de Recherches sur la Jeunesse de la Faculté Latino-Américaine de Sciences Sociales et boursière postdoctorale du Conseil National de Recherches Scientifiques et Techniques. Code postal: Olivieri 212 PA, Ville autonome de Buenos Aires. Email: acorica@flacso.org.ar 


\section{Introducción}

La población argentina joven ha logrado obtener en las últimas décadas mayores niveles educativos que en periodos anteriores. La expansión del sistema educativo ha sido notoria no solo en cuanto al número de personas que asisten (expansión horizontal), sino también a la cantidad de establecimientos estudiantiles habilitados (expansión vertical) ${ }^{1}$. Los signos de este avance se corresponden con un crecimiento de la escolarización, una mayor inclusión educativa y una prolongación del periodo escolar obligatorio, datos que se reflejan en las cifras censales en el largo plazo.

Tomando en cuenta a los adolescentes entre 15 y 19 años de edad, en 1970 asistía a algún establecimiento educativo el 35 \%; 20 años más tarde, en 1991, el 54,3 \%, y en 2010, el 69,3\%. Dicho crecimiento fue producto, en gran parte, de la incorporación de los grupos menos favorecidos - desde el punto de vista económico- de la población. Son estos jóvenes los que ingresan mayoritariamente en el nivel medio de enseñanza, o secundaria, que hoy en día permanece más años dentro del sistema educativo, a tal punto que se incrementa de forma notable el porcentaje de jóvenes que logran terminar y acceder a la educación superior (Córica y Otero, 2015).

En paralelo, se observa un incremento del nivel educativo de los jóvenes entre 20 y 24 años de edad. En esa franja, en 1970, solo el 20,3 \% había completado la secundaria, mientras que en 2010, el porcentaje llegó al 50,2 \% (Censo Nacional de Población del Instituto Nacional de Estadística y Censos [INDEC], 19702010). Finalmente, en el caso de los jóvenes entre 25 y 29 años (jóvenes adultos), en 1970, el 15,8 \% obtuvo el título de la secundaria, y solo el 2,8 \% completó estudios de nivel superior, mientras que en el censo de 2010, el 45,3 \% de estos jóvenes completaron la secundaria, y el 12,8 \%, carreras universitarias y terciarias (carreras técnicas y docentes).

Como contracara, los logros educativos encierran renovadas preguntas acerca de los alcances y obstáculos de la expansión, así como sobre la necesidad de adecuaciones en intervenciones orientadas a la inclusión educativa. Entre otros, surgen interrogantes acerca de los grupos poblacionales más rezagados en materia de escolarización y de cuáles han sido los avances en términos de inclusión en torno a la evolución de cada uno de los niveles educativos. Tomando en cuenta el avance de la escolarización de la población joven en el país durante el último periodo, en este artículo se abordarán los diferenciales de lo sucedido en la escolarización media y la escolarización superior en la franja poblacional de jóvenes, en particular en la última década.

Se presenta un análisis estadístico y documental con base en fuentes secundarias de información: Encuesta Permanente de Hogares (EPH) del Instituto

Utilizaremos indistintamente los términos nivel medio y escuela secundaria. 
Nacional de Estadística y Censos (INDEC), del 2. ${ }^{\circ}$ trimestre de 2006 y de 2012²; reconstrucciones provenientes de las bases de datos del Sistema de Información de Tendencias Educativas en América Latina (SITEAL), del Instituto Internacional de Planeamiento de la Educación (IIPE) Organización de las Naciones Unidas para la Educación, la Ciencia y la Cultura (Unesco, por sus siglas en inglés), y de la Organización de Estados Iberoamericanos (OEI), del periodo 1993-2012; las normativas vigentes, documentos de programas nacionales y políticas educativas orientadas a la inclusión ${ }^{3}$.

La primera parte del texto delinea el esquema del sistema educativo argentino y presenta información relevante sobre las transformaciones normativas y políticas de las últimas décadas, tomando en cuenta las estrategias de inclusión y la expansión educativa. La segunda parte expone el análisis estadístico de la población joven en torno a la asistencia y los logros en el sistema educativo, particularmente en el nivel secundario. La tercera parte profundiza en las tendencias respecto a la educación superior a lo largo del periodo de referencia, y las conclusiones retoman las preguntas centrales que se desprenden del análisis.

\section{Panorama general del sistema educativo argentino}

La estructura del sistema educativo argentino ha sufrido modificaciones sustantivas a lo largo de la década de los noventa. Originalmente, estuvo organizada en: primaria, por un periodo de siete años (orientada a estudiantes entre 6 y 12 años de edad); secundaria, por un periodo de cinco años (orientado a estudiantes entre 13 y 18 años), y superior (terciaria y universitaria). Mientras se mantuvo vigente dicha estructura, solo la primaria era obligatoria.

A partir de la aplicación de la Ley Federal de Educación 24195, sancionada en 1993, el sistema sufrió modificaciones. Esta ley definió una nueva estructura, organizada en un nivel inicial (de un año), tres ciclos de educación general básica (de nueve años), polimodal (de tres años) y educación superior. Debido a esta reforma, se extendió la obligatoriedad a diez años de escolarización, que comprenden el nivel inicial y la educación general básica. No obstante, la estructura volvió a cambiar con la sanción de una nueva Ley de Educación Nacional n. 26.206/06. Esta medida introdujo transformaciones en el marco normativo y en las metas educativas para los próximos años, como redefinir la culminación de la secundaria y extender la obligatoriedad a doce años.

2 Este texto toma como antecedente parte del trabajo desarrollado en el proyecto n. ${ }^{\circ} 10 / 2013$, realizado con el apoyo del Programa de Internalización de la Educación Superior y Cooperación Internacional, Núcleo de Estudios e Investigaciones en Educación Superior del Sector Educativo del Mercosur, por medio de un convenio de cooperación entre la Facultad Latinoamericana de Ciencias Sociales (Flacso, Argentina), la Universidad de la República Oriental del Uruguay (Udelar) y la Universidad Federal de São Carlos (UFSCar).

3 Parte de las ideas expresadas en el presente texto retoman planteamientos esbozados en Otero, A. y Córica, A. (2017). Jóvenes y educación superior en Argentina. Evolución y tendencias. Revista Interamericana de Educación de Adultos, $39(1), 11-28$. 
Es decir, que las modificaciones en el marco legislativo han acompasado la ampliación. En la primaria, ya en 1993, la obligatoriedad se estableció con la Ley Federal de Educación. Más recientemente, se estableció la obligatoriedad de la secundaria, mediante el artículo 16 de la Ley de Educación, y durante 2015, la obligatoriedad de la educación inicial para niños de cuatro años (BO.07/01/15, N³3044).

Asimismo, desde mediados de 2000 se instituyó un avance significativo en materia económica, pues la Ley de Financiamiento Educativo (Ley 26.075 de 2006) logró establecer un aumento progresivo de la inversión en educación, ciencia y tecnología, con una asignación del 6,47 \% del producto interno bruto (PIB) para el área educativa general, y la duplicación del presupuesto universitario para el periodo 2002-2012, en función de la participación en el PIB, según estimaciones realizadas por la Secretaría de Políticas Universitarias (SPU) ${ }^{4}$.

La expansión en la cobertura del sistema educativo tuvo que ver con distintos factores que intervinieron en este proceso, entre otros, la mencionada ampliación de la obligatoriedad, la expansión de la oferta educativa y distintas acciones orientadas a fomentar la inclusión educativa y a estimular el incremento de la escolarización. Los cambios legislativos de mediados de 2000 estuvieron acompañados en los años siguientes por un conjunto de políticas sociales. Desde el punto de vista de la política pública, se han impulsado distintas intervenciones desde el Estado nacional para la promoción de la inserción de niños y jóvenes, y para apoyar el acompañamiento de las trayectorias escolares de los estudiantes provenientes de hogares de sectores bajos. En esta línea, destacan las becas o las transferencias condicionadas de ingresos y el desarrollo de programas de reingreso a la escuela.

Junto a la aplicación de controles sobre el trabajo infantil, comenzó la implementación de la asignación universal por hijo, a través del Decreto 1602 de 2009. Esta asignación universal está dirigida a todos los menores de 18 años cuyos padres o tutores se encuentran desocupados, con un trabajo informal, laborando por cuenta propia o haciendo parte del servicio doméstico, y que perciban remuneraciones inferiores al salario mínimo vital y móvil. Se trata de un seguro social y una transferencia de ingresos ligada a contraprestaciones destinada a la asistencia escolar de los menores, el control de la salud para la primera infancia y el cumplimiento del calendario de vacunación obligatorio. Entre todas las medidas, es la de mayor impacto, por cobertura y población beneficiada, con una amplia legitimidad, por su efectividad y alcances. Para marzo de 2016, los beneficiarios de la asignación universal por hijo sumaban un total de 3.798 .494 (mujeres y varones) ${ }^{5}$.

4 Datos disponibles en: http://portales.educacion.gov.ar/spu/

5 Datos disponibles en http://observatorio.anses.gob.ar/archivos/publicaciones/PPT\%20-\%20AUH $\% 201 \% 20$ C $\% 20$ 2016\%20G_v99.pdf 
Ahora bien, en 2006 el sistema educativo vuelve a modificarse: la primaria pasa a durar seis años y la secundaria también. Además, la Ley de Educación de 2006 establece la obligatoriedad del nivel secundario y se impulsan distintas modalidades de programas destinados a la inclusión educativa. Además, durante 2008 se comenzó a implementar el Plan de Finalización de Estudios Primarios y Secundarios (FINES) ${ }^{6}$, con miras a posibilitar que jóvenes y adultos mayores de 18 años finalicen la escolaridad obligatoria. Esta medida propone resignificar el diseño de las propuestas educativas a través de formatos más flexibles (como aprendizajes autónomos, contextualizados y por módulos); prevé el acompañamiento a través de dispositivos (como teleclases y tutores docentes), y rompe con los límites espaciales tradicionales, pues no solo se implementa en escuelas secundarias, sino también en fundaciones, sindicatos, asociaciones de fomento y otras entidades. De este modo, ofrece una propuesta con un formato más flexible que el tradicional secundario (Terigi, 2008).

En materia de política educativa en educación superior, las intervenciones, desde el Estado nacional, han tenido menos prioridad que el resto de los niveles educativos. Se estableció la continuidad del Programa Nacional de Becas Universitarias (PNBU) ${ }^{7}$, por medio de la Resolución 464/96 - el de más larga data - , actualmente dirigido a alumnos de carreras de grado pertenecientes a las ramas de la salud, las humanidades y las ciencias sociales. A finales de 2000, se implementó el Programa Nacional de Becas Bicentenario (PNBB) ${ }^{8}$, mediante el Decreto (PE) 99/09, que cubre las carreras vinculadas a las ciencias aplicadas, las naturales, las exactas y las básicas, consideradas estratégicas para el desarrollo económico y productivo del país. Por último, el Programa Nacional de Becas para Carreras de Grado en el Área de las Tecnologías de la Información y la Comunicación (PNBTICS), por medio de la Resolución 2031/08, un sistema de becas diseñado para fortalecer y promover el incremento de la matrícula de estudiantes en las carreras relacionadas con las tecnologías de la información y la comunicación (TIC).

Pese a las medidas, tanto por la cantidad de adjudicaciones, como por los montos, estos programas de becas tienen un bajo alcance. Según algunos Autores, suponen una política inclusiva para los jóvenes con menos recursos económicos, pero cubren bajos porcentajes en función de las matrículas en universidades nacionales (Chiroleu, 2013) ${ }^{9}$.

6 Es un plan específico creado a término y refrendado por los ministros de educación del país, a través de las resoluciones 22/07 y 66/08 del Consejo Federal de Educación. La medida es de alcance nacional y funciona en todas las jurisdicciones. Está dirigida a jóvenes que adeudan materias de secundaria y a aquellos que no iniciaron o no terminaron la primaria o la secundaria.

7 Becas otorgadas desde mediados de los noventa, dirigidas a estudiantes de universidades nacionales provenientes de hogares en situación de pobreza estructural. A partir de 2000 , incluyen una ayuda a estudiantes indígenas y discapacitados. El monto es de 95 dólares.

8 Becas otorgadas a ingresantes o cursantes en carreras de grado, técnicas y universitarias, no universitarias y profesorados terciarios, vinculados a ingenierías, ciencias aplicadas, ciencias naturales, ciencias exactas, ciencias básicas y enfermería. El monto mensual es de 184 dólares.

9 Según el Anuario de Estadísticas de la Secretaría de Políticas Universitarias (2011), considerando los tres programas nacionales, las becas adjudicadas eran de 48.052 dólares, que abarcaban una porción ínfima del estudiantado del nivel (22.535.859 alumnos matriculados en educación superior. 
Si bien esta serie de medidas contribuyó a dar lugar a un marco de derechos que aún se encuentra en proceso de evolución, crea un nuevo entorno para pensar en acciones de promoción de las condiciones de vida de la juventud y sus ámbitos de socialización e integración ${ }^{10}$.

\section{La expansión educativa en cifras, interrogantes y reflexiones}

Al igual que para otros países de la región, en Argentina los niveles de escolaridad obligatoria definida por las leyes no se cumplen en la práctica, y el peso de las desigualdades sigue vigente. El origen social, la adscripción étnica, la diferencia urbano-rural, entre otros, continúan siendo determinantes de las desigualdades en la participación y logros educativos. Sin embargo, la incorporación masiva de nuevos estudiantes al sistema educativo es materia de discusión en las ciencias sociales y en la agenda de política educativa. Peters (2014, p. 188) sostiene que estas desigualdades se están desplazando progresivamente hacia los niveles más altos del sistema educativo, pasando de la primaria-secundaria hacia los últimos años de la secundaria y niveles superiores. Como dice el autor, "se está desplazando la función de filtro social de los sistemas educativos, aunque el mecanismo en sí mismo se mantiene mayormente inalterado".

Asimismo, otros autores argumentan que la tasa de expansión educativa se ha ido desacelerando, dando pie a pensar que, si bien los niveles iniciales (jardín, pre-escolar y primaria) alcanzan a la universalización ${ }^{11}$, este logro dista de ser un objetivo alcanzable en el corto plazo para la secundaria (Kessler, 2014).

Ahora bien, este proceso de desaceleración no se corrobora de igual forma para el caso de la educación superior. La evolución de las tasas de escolaridad e indicadores educativos reflejan el paulatino incremento de la cobertura educativa desde finales del siglo XX. Si para 1993 la tasa de escolarización era del 71,2 \%, para el total de la población en 2012 ascendía al 77,6 \% (figura 1). Considerado en su conjunto, el incremento da cuenta no solo de una mayor incorporación de la población al sistema escolar en los niveles inicial y medio, sino también de un incremento sostenido en el acceso a la educación superior.

10 Más recientemente, se suma a las medidas el Programa de Respaldo a Estudiantes de Argentina Progresar, creado mediante el Decreto (PE) 84/2014 (BO 27/01/2014), destinado a jóvenes entre 18 y 24 años que no terminaron los estudios obligatorios y que están desocupados o trabajan en empleos precarios. Véase el enlace https://www.llyasoc. com/5025/previsional-polticas-sociales-creacin-del-programa-de-respaldo-a-estudiantes-argentinos---progresar.html 
Figura 1. Tasa de escolarización del total de la población. Argentina, 1993-2012

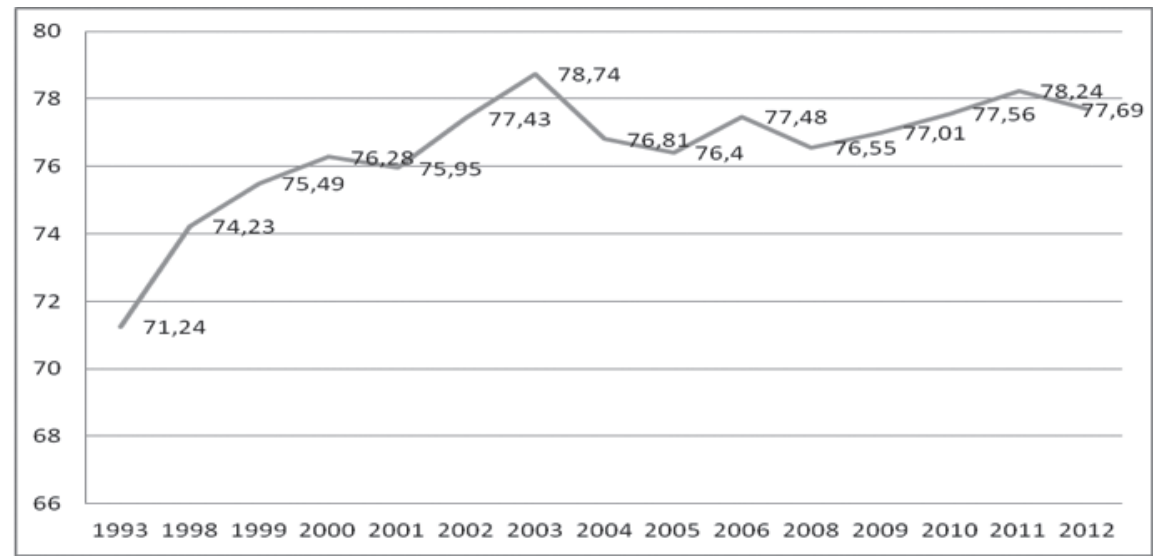

Fuente: Instituto Internacional de Planeamiento de la Educación - Organización de las Naciones Unidas para la Educación, la Ciencia y la Cultura; Organización de Estados Iberoamericanos. Con base en la

Encuesta Permanente de Hogares del Instituto Nacional de Estadística y Censos, Argentina.

Al desagregar el incremento a través del análisis de los datos de la Encuesta Permanente de Hogares (EPH) que realiza el Instituto Nacional de Estadística y Censos (INDEC), específicamente aquellos correspondientes al segundo trimestre de 2006 y 2012, la participación educativa de la población joven para dichos años se percibe por un incremento de la asistencia escolar de jóvenes entre 18 y 24 años, una variación positiva (un aumento) del 1,6 \% entre 2006 y 2012. De este modo, cerca del 50 \% de la población de dicho grupo se encuentra actualmente en el sistema educativo.

Tabla 1. Porcentaje de la población de 15 a 29 años* que asiste a un establecimiento educativo, 2006-2012

\begin{tabular}{|l|c|c|c|}
\hline \multirow{2}{*}{ Grupos de edad } & \multicolumn{2}{|c|}{ Año } & Variación \\
\cline { 2 - 3 } & $\mathbf{2 0 0 6}$ & $\mathbf{2 0 1 2}$ & $\mathbf{2 0 1 2}$ \\
\hline 15 a 17 años & 38,56 & 37,73 & 1,6 \\
\hline 18 a 24 años & 47,41 & 49,09 & 1,6 \\
\hline 25 a 29 años & 14,02 & 13,17 & 0,9 \\
\hline \multicolumn{3}{|c|}{ * Por grupos de edad. }
\end{tabular}

Fuente: elaboración propia con base en la Encuesta Permanente de Hogares del Instituto Nacional de Estadística y Censos, Argentina, 2006 y 2012, segundo trimestre.

Al comparar los porcentajes en el periodo de análisis, se observa un incremento en la asistencia escolar entre la población de 18 a 24 años y una mayor participación de los distintos quintiles de ingresos, indicando que se extienden los años 
de permanencia en el sistema educativo y se incrementa la inserción educativa de los grupos sociales de más bajos ingresos.

Ahora bien, este incremento en la asistencia educativa no se da de igual forma en el grupo de jóvenes de 25 a 29 años, concentrándose el incremento en los quintiles de mayores ingresos. (tabla 2).

Tabla 2. Porcentaje de la población de 15 a 29 años* que asiste a un establecimiento educativo, 2006-2012

\begin{tabular}{|l|c|c|c|c|c|c|c|c|c|c|}
\hline \multirow{2}{*}{$\begin{array}{l}\text { Grupos de } \\
\text { edad }\end{array}$} & \multicolumn{2}{|c|}{ Quintil 1 } & \multicolumn{2}{c|}{ Quintil 2 } & \multicolumn{2}{c|}{ Quintil 3 } & \multicolumn{2}{c|}{ Quintil 4 } & \multicolumn{2}{c|}{ Quintil 5 } \\
\cline { 2 - 12 } & 2006 & 2012 & 2006 & 2012 & 2006 & 2012 & 2006 & 2012 & 2006 & 2012 \\
\hline 15 a 17 años & 54,58 & 50,24 & 40,26 & 38,23 & 32,16 & 31,67 & 26,91 & 27,02 & 23,17 & 22,26 \\
\hline 18 a 24 años & 38,13 & 43,69 & 47,84 & 49,71 & 53,85 & 54,29 & 53,15 & 53,01 & 51,03 & 49,3 \\
\hline 25 a 29 años & 7,28 & 6,06 & 11,89 & 12,06 & 13,99 & 14,04 & 19,94 & 19,96 & 25,79 & 28,44 \\
\hline
\end{tabular}

* Por grupo de edad y nivel de ingreso.

Fuente: elaboración propia con base en la Encuesta Permanente de Hogares del Instituto Nacional de Estadística y Censos, Argentina, 2006 y 2012, segundo trimestre.

Según los datos analizados, aunque las condiciones de acceso a mayores niveles educativos en los distintos grupos sociales han mejorado, aún falta optimizar la permanencia de los jóvenes de los quintiles más bajos.

\section{Jóvenes y educación secundaria}

Como se señaló líneas atrás, la masificación de la escolaridad en las últimas décadas va de la mano de la expansión de la secundaria. En la última década, dicha expansión estuvo acompañada de la extensión de la obligatoriedad de este nivel de enseñanza, con el objetivo principal de incorporar sectores sociales tradicionalmente excluidos del nivel medio.

Haciendo un breve recuento histórico, en la década de los setenta comenzó la expansión de la secundaria. Los principales beneficiarios fueron los sectores medios, que comenzaron a crecer en proporción directa a las posibilidades del acceso a la educación (Finkel, 1977). A finales de los ochenta y principios de los noventa, alumnos de los sectores más pobres, que aún quedaban excluidos de la educación media, se incorporaron; la investigación educativa conceptualizó esta incorporación como "masificación de la enseñanza secundaria", que se convirtió en fruto de numerosos análisis (Tenti Fanfani, 2008). No obstante, algunas investigaciones realizadas a finales de los ochenta señalan que el sistema educativo argentino estaba segmentado y que, por lo tanto, el desplazamiento 
y la movilidad social a través de la educación se estaban perdiendo. Según estos estudios, la certificación ya no garantizaba un buen trabajo, solo tendía a reforzar la posición social de origen. Esta tendencia se profundizó aún más con las transformaciones que tuvieron lugar en la década de los noventa y con la crisis de 2001, hasta lograr que la idea de ascenso social a través de la educación terminara por desvanecerse.

Durante los noventa, los estudios comenzaron a revelar que en el sistema educativo argentino no solo se había profundizado la diferenciación social, es decir, la segmentación educativa, sino que, a su vez, se había producido una fragmentación entre los segmentos. El sistema comenzó a organizarse alrededor de patrones culturales que no reconocían elementos de continuidad entre unos y otros, y esto produjo que las escuelas dejaran de ser espacios sociales y culturalmente homogéneos para convertirse en un contexto de desigualdad y diversidad. Cada escuela pasó a constituir un fragmento que selecciona e incorpora a un grupo social particular. La selección se hace por la participación en diferentes circuitos educativos, y el ingreso a aquellos está asociado al capital económico, social y cultural que posee un grupo cada vez más reducido de la población (Tiramonti, 2004). La fragmentación identificada en los estudios en la década de los noventa da cuenta de que la diferenciación en el sistema educativo no solo se da por cierta condición económica y social, sino también por patrones culturales, lo cual profundiza la diferenciación entre los grupos.

La expansión y masificación que se da en este nivel educativo se expresa en los datos de la evolución de la tasa neta de escolarización secundaria (figura 2), dan cuenta de la consolidación de la incorporación de más del 80 \% de la población que ha asistido al nivel medio de enseñanza en la última década.

Figura 2. Tasa neta de escolarización secundaria del total de la población. Argentina, 2000-2012

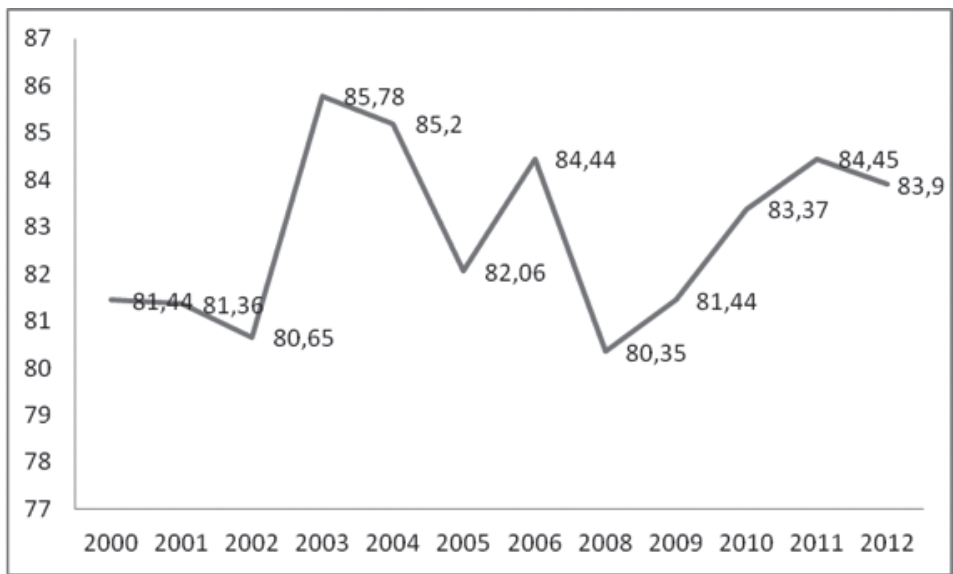

Fuente: Instituto Internacional de Planeamiento de la Educación - Organización de las Naciones Unidas para la Educación, la Ciencia y la Cultura; Organización de Estados Iberoamericanos. Con base en la Encuesta Permanente de Hogares del Instituto Nacional de Estadística y Censos, Argentina. 
Cabe señalar que este periodo se dio en el contexto de un conjunto de intervenciones estatales que habrían contribuido a que los jóvenes que estaban fuera del sistema educativo se reincorporaran. Así, el panorama estaría indicando que la conjunción de cierto mejoramiento en las condiciones sociales y económicas, más las políticas implementadas, proveen las condiciones para que los jóvenes se reinserten en el sistema educativo o continúen estudiando. Para unos, la mejora significó terminar sus estudios secundarios; para otros, continuar carreras de nivel superior. Por lo tanto, los datos reflejan una mejora favorable para la población joven en cuanto a la posibilidad de alcanzar mayores niveles educativos, y esto demuestra la efectividad de la batería de las políticas orientadas a la inclusión educativa, especialmente las vinculadas con la finalización de los estudios secundarios.

Asimismo, independientemente del sector social, el porcentaje de jóvenes mayores de 20 años que completó la educación secundaria aumentó durante el periodo 1998-2012 (figura 3). Entre los jóvenes con menores ingresos, este incremento fue mayor; el porcentaje que terminó la secundaria en 1998 fue del $10,28 \%$, pero en 2012 ascendió al 25,2\%. Por su parte, entre los sectores medios y altos, el 20 \% obtuvo el título de nivel medio en 1998, y el 25 \%, en 2012.

Figura 3. Porcentaje de jóvenes mayores de 20 años que culminó la secundaria en el periodo 1998-2012 por nivel de ingresos

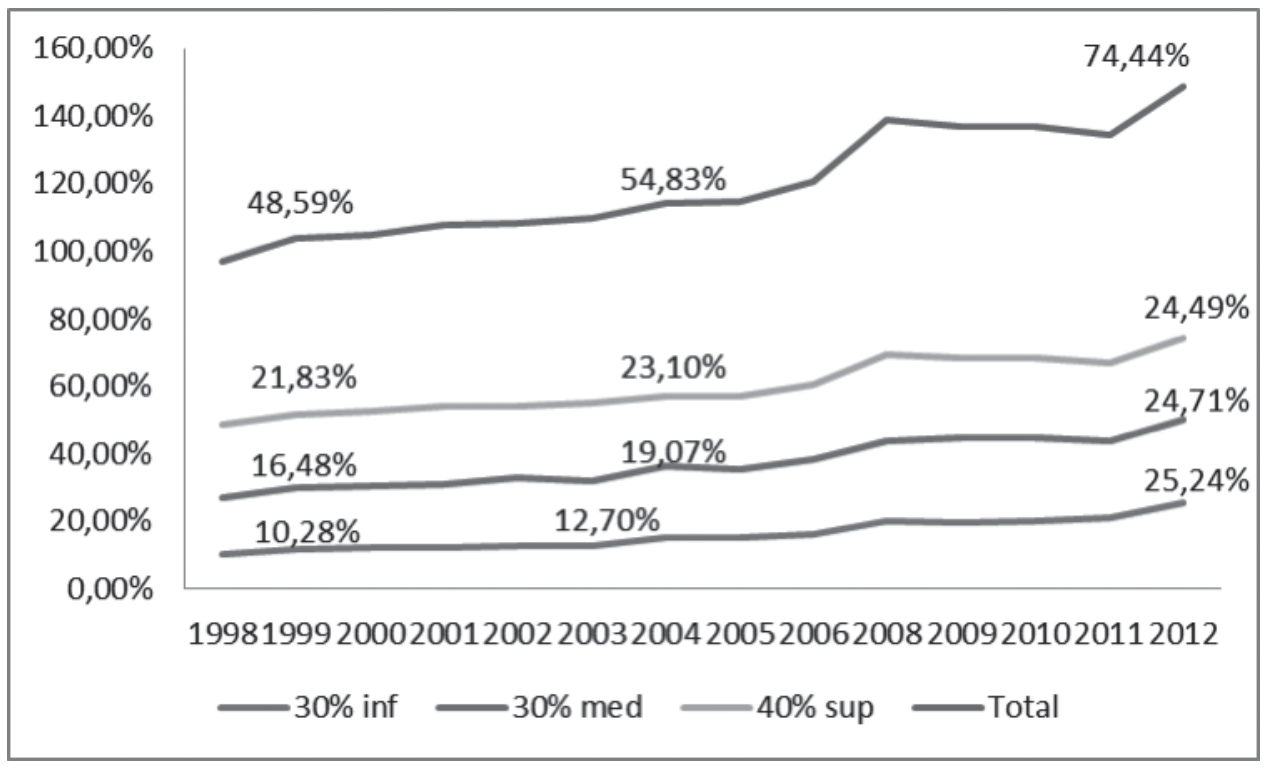

Fuente: Instituto Internacional de Planeamiento de la Educación, Organización de las Naciones Unidas para la Educación, la Ciencia y la Cultura; Organización de Estados Iberoamericanos. Con base en la Encuesta Permanente de Hogares del Instituto Nacional de Estadística y Censos, Argentina.

Como efecto del avance de la escolarización por una mayor población de egresados de la secundaria, la matrícula de la educación superior ha crecido a un 
ritmo elevado y, como se verá, sigue haciéndolo en el periodo analizado. Ambos niveles educativos confluyeron en el incremento de la población que está en condiciones de acceder a la educación superior después de obtener el título de nivel medio. Si bien la demanda social por la educación superior dista de ser un fenómeno reciente, continúa su curso.

Una de las hipótesis que se consideran a partir de los datos procesados es que a medida que se amplían las oportunidades de culminar la secundaria, es mayor la demanda de educación superior. Y a su vez, esta hipótesis podría ser reforzada al considerar la ampliación de la red institucional de la oferta pública y privada del nivel.

En el siguiente apartado se analiza la participación de la población de jóvenes argentinos en la educación superior y se señalan las tendencias más destacadas en relación con la expansión y la composición.

\section{Jóvenes y educación superior}

La educación superior en Argentina se ha destacado por su carácter predominantemente público, gratuito y de ingreso irrestricto ${ }^{12}$. Está integrada por servicios educativos de gestión estatal y privada, gestión cooperativa y gestión social, con una estructura binaria donde se distinguen dos subsistemas educativos: el Sistema Universitario Nacional, conformado por universidades e institutos universitarios, y el Sistema de Educación Superior no Universitaria, que reúne institutos de educación superior ${ }^{13}$.

Algunos expertos afirman que el sistema universitario fue el nivel educativo que más se desarrolló a lo largo de estos treinta años de democracia en el país. Ya desde los ochenta se verifica un fenómeno de masificación, con un crecimiento de la matrícula, así como una ampliación sustantiva de la cantidad de instituciones de gestión pública y privada.

El nivel superior se destaca por su tendencia a configurarse como una red donde predomina el peso de establecimientos públicos, sobre todo de universidades grandes y tradicionales, que concentran la mayor parte de la matrícula del nivel. Junto con las universidades fundacionales, como la Universidad de Córdoba (1613) y la Universidad de Buenos Aires (1821), desde finales del siglo pasado se observa una acelerada multiplicación de establecimientos del

12 El acceso está mediado por las regulaciones de las propias instituciones de educación superior (se trata de un sistema mixto donde conviven básicamente el ingreso directo, exámenes, cursos de ingresos, etc.), y como condiciones básicas de ingreso se requiere la aprobación del nivel de enseñanza medio, aunque con el último cambio legislativo ((Ley de Educación Nacional, artículo 7)), se admite el ingreso de personas mayores de 25 años de edad que, sin contar con el título medio, aprueben una evaluación (establecida por las mismas instituciones de educación) a través de la cual se acrediten sus condiciones para comenzar estudios superiores. 
circuito universitario, en lo que se refiere a la educación terciaria no universitaria (Krotsch, 2001; García de Fanelli, 2006).

Desde una perspectiva histórica, y teniendo en cuenta solo las universidades, la cantidad de instituciones fue creciendo en sucesivas oleadas. A las siete universidades públicas (hasta 1956), se agregaron una veintena de establecimientos. A partir de entonces, y hasta 1990, se crearon diecinueve universidades públicas más y doce universidades privadas (Mollis, 2008). Luego - con un claro sesgo privatista - entre 1990 y 2000, se crearon veintiséis universidades de gestión privada. Y, desde 1989 hasta la actualidad, se impulsó la creación de nuevas universidades públicas, parte de las cuales se ubican en el territorio del Conurbano Bonaerense.

A finales de 2000, y principios de la nueva década, se observó un nuevo impulso del Estado con la creación de nuevas universidades e institutos universitarios ${ }^{14}$. Esta última etapa fecha un cambio respecto a la política de la década de los noventa, a partir de la cual el Estado asumiría un papel más importante en el crecimiento de la red institucional. Con base en los datos de la Secretaría de Políticas Universitarias (SPU), en el periodo 2003-2015 se crearon veinticuatro instituciones de gestión estatal y trece de gestión privada.

Esta estrategia de expansión reconfigura el mapa de distribución de acceso e instala una nueva posibilidad de incrementar la cobertura. Y, en términos de los potenciales estudiantes, establece un nuevo camino de entrada, jamás pensado dos o tres décadas atrás.

En cuanto a la expansión educativa que se ha venido señalando, se observa que efectivamente el acceso a una carrera universitaria es uno de los grandes logros de la última década. Esto se verifica tanto en el crecimiento de la matrícula en este nivel de enseñanza, como en la creación de más instituciones universitarias, lo que favorece a la continuidad educativa.

La evolución en relación con la población de egresados contrarresta y relativiza las estimaciones acerca del alcance real del avance en función de permanencia y terminación del ciclo para el conjunto de los ingresantes. La contracara de las cifras alentadoras, la ampliación de las instituciones educativas y las tendencias respecto a la finalización muestran que las trayectorias educativas no siempre concluyen de forma exitosa.

Ahora bien, aunque la permanencia y escasas cifras de finalización del nivel siguen siendo un problema clave en la performance del sistema de educación superior argentino, según los datos procesados a través de la base disponible en el Instituto Internacional de Planeamiento de la Educación, se destaca que

14 En esta línea, se crean varias universidades, como es el caso de la Universidad Nacional de Avellaneda (Ley 26543 de 2009), la Universidad Nacional Arturo Jauretche en Florencio Varela (Ley 26.576 2009), la Universidad Nacional de Moreno (Ley 26575 de 2009), la Universidad Nacional de José Clemente Paz (Ley 26577 de 2009) y la Universidad Nacional del Oeste en Merlo (Ley 26544 de 2009), entre otras. 
de la población mayor de 25 años, cada vez son más las personas que obtienen un título superior o universitario: del $12,7 \%$, en 2000, pasó a casi el $20 \%$, en 2012 (figura 4).

Figura 4. Porcentaje de la población de 25 años y más con educación superior o universitaria completa. Argentina, 2000-2012

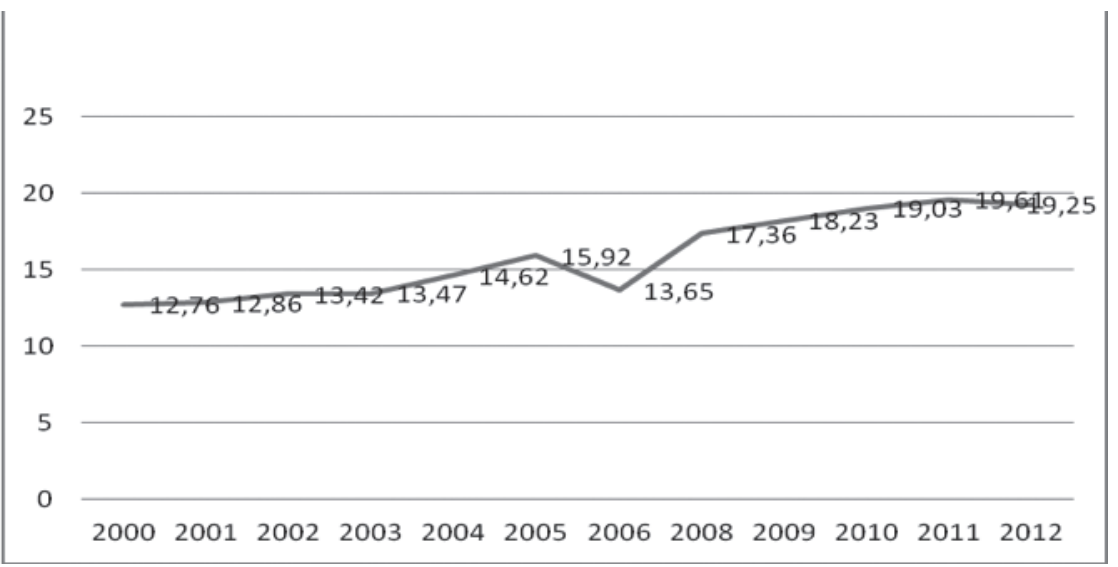

Fuente: Instituto Internacional de Planeamiento de la Educación - Organización de las Naciones Unidas para la Educación, la Ciencia y la Cultura; Organización de Estados Iberoamericanos. Con base en la Encuesta Permanente de Hogares del Instituto Nacional de Estadística y Censos, Argentina.

La obtención de un título de nivel superior se observa en todos los grupos sociales, pues se registra un incremento de casi el $10 \%$. Si bien en los sectores de escasos recursos este aumento es menor, es importante destacar su incremento en la obtención de un título de nivel superior (en el periodo 2000-2012 fue del $5 \%$ ), como se observa en la figura 5 .

A la par con la extensión de la cobertura de la educación media, los sesgos que persisten por causas socioeconómicas operan como límite para una mayor inclusión de los jóvenes en la educación superior. Las exclusiones dadas en el nivel previo generan un obstáculo para el acceso al sistema educativo superior, que, por sus características de gratuidad y; de acceso irrestricto, ofrece condiciones óptimas.

La demanda potencial que supone la expansión de la matrícula media (luego de establecida su obligatoriedad) queda relativizada por el fenómeno de la deserción o abandono. Incluso entre quienes obtienen el título de la secundaria, los déficits en las habilidades para enfrentar los estudios superiores suponen un obstáculo para sacarlos adelante.

El paso de la secundaria a los estudios superiores ha sido históricamente conflictivo. Puesto que los cuestionamientos apuntan a la escasa articulación entre los distintos niveles del sistema educativo argentino, se desarrollaron programas dirigidos a remover los obstáculos generados por esta tensión. La 
Figura 5. Porcentaje de la población de 25 años y más con educación superior o universitaria completa. Argentina, 1998-2012. Por nivel de ingresos.

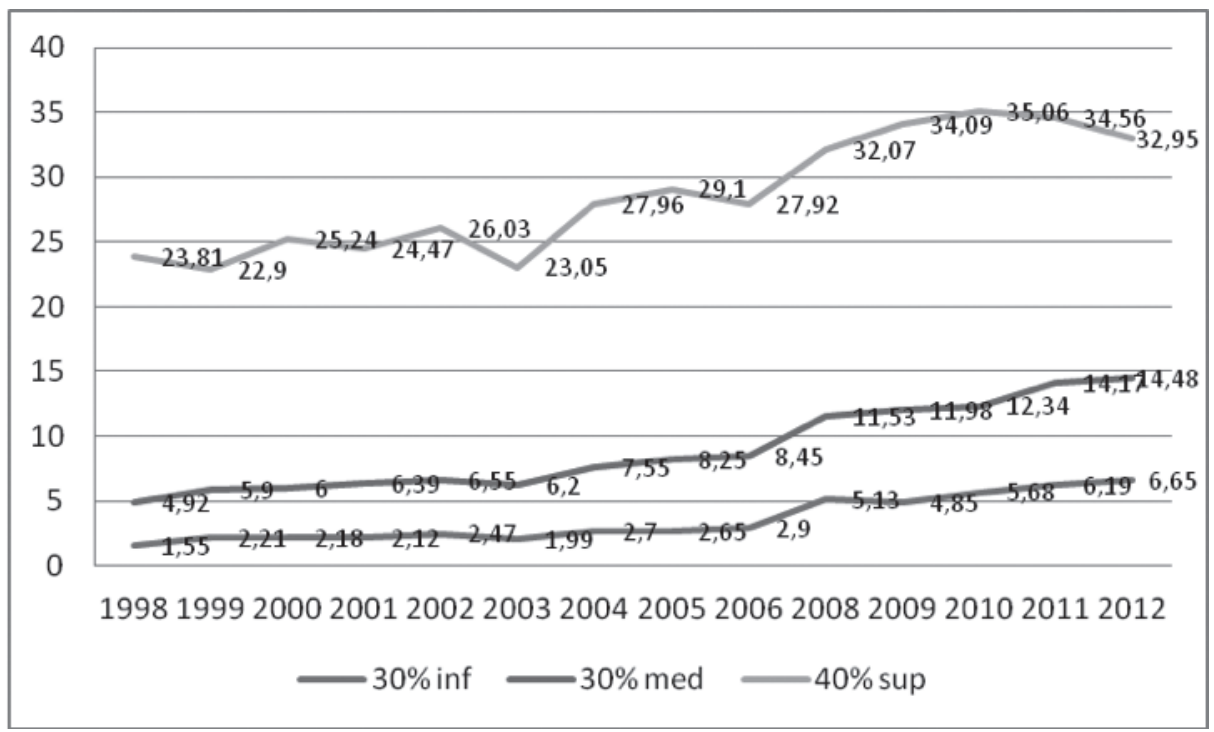

Fuente: Instituto Internacional de Planeamiento de la Educación - Organización de las Naciones Unidas para la Educación, la Ciencia y la Cultura; Organización de Estados Iberoamericanos. Con base en la Encuesta Permanente de Hogares del Instituto Nacional de Estadística y Censos, Argentina.

conexión entre las universidades y las secundarias implicó el desarrollo de dos tipos de propuestas: unas destinadas a los docentes de ambos niveles y a los estudiantes de los últimos años de la enseñanza secundaria, y otras destinadas a fortalecer o a diseñar estrategias pedagógicas e institucionales para la mejora del desempeño académico de los recientes ingresantes. Las universidades han desarrollado distintas experiencias con el objetivo de aportar soluciones a los problemas de abandono o ralentización de los estudios superiores (Araujo, 2009). Resta decir que la segmentación y la precaria calidad educativa de la escuela secundaria es una cuestión de peso a la hora de explicar el alto desgranamiento dado en los primeros años de los estudios superiores.

Las discusiones sobre los alcances de la democratización en cuanto al acceso y al ciclo de educación superior son un terreno de prolíferos debates en el plano local (Gessaghi y Llinás, 2005). Se han acuñado términos como inclusión con calidad (Araujo, 2009), inclusión o democratización (Chiroleu, 2013) e inclusión excluyente (Ezcurra, 2013), todos ellos dados en el ámbito de una tensión nodal entre masividad y calidad. En paralelo, un aspecto central es la convivencia entre la ampliación del acceso, con escasos porcentajes de graduación de la educación superior (García de Fanelli, 2006).

Ezcurra sostiene que estamos ante un proceso de "inclusión excluyente", porque si bien se observa un incremento significativo de la matrícula en educación superior, este convive con el fenómeno del abandono, sobre todo en los primeros años de ingreso al sistema, por parte de la población más desfavorecida en la 
distribución del capital económico y cultural. Esto significa que el acceso a la educación superior no es garantía de inclusión con permanencia y graduación del nivel (Ezcurra, 2013, p. 9).

Al analizar los resultados segmentados por nivel socioeconómico, puede observarse, con base en la distribución por quintiles de ingresos, un incremento en la asistencia escolar entre los jóvenes de diferentes estratos socioeconómicos y una mayor asistencia a establecimientos terciarios que a la universidad.

La educación terciaria atrae en una mayor proporción a jóvenes de estrato socioeconómico más bajo, debido a múltiples factores: corta duración y rápida salida laboral: menores exigencias y formatos pedagógicos más estandarizados que en las universidades; mayor distribución regional de la oferta de establecimientos terciarios; posibilidades de compatibilizar tiempos de estudio y trabajo, entre otros.

El deterioro de la calidad de la educación media, verificado en las últimas décadas, es un agregado de peso a esta inclinación, porque da cuenta de la desigualdad de oportunidades para acceder a la educación universitaria frente a los jóvenes de clase media y media-alta.

Esta tendencia marcha en dirección similar a los argumentos expuestos en investigaciones acerca de la situación del nivel terciario en países de América Latina, incluyendo el caso de Argentina. En un estudio reciente, se afirma la relevancia del nivel terciario en la educación superior argentina, junto con el papel que cumple para el acceso de los jóvenes de niveles socioeconómicos bajo y medio bajo. Según el estudio, en el contexto de expansión educativa, donde los diplomas de la secundaria se instalan como requisitos de acceso al empleo, y donde impera una demanda social cada vez mayor por la obtención de titulaciones de educación superior, los establecimientos terciarios ofrecen una alternativa para quienes aspiran a continuar estudiando, pero que no logran acceder a la universidad, sea por los costos que demanda una carrera universitaria, por necesidades, o por una inclinación hacia carreras cortas con perspectivas de ingreso al mercado laboral en un menor tiempo (García de Fanelli y Jacinto, 2010, p. 74).

Tabla 3. Porcentaje de la población de 18 a 29 años que finalizó el nivel superior según los quintiles de ingresos, 2006-2012

\begin{tabular}{|l|c|c|c|c|c|c|c|c|c|c|}
\hline & \multicolumn{4}{|c|}{2006} & \multicolumn{5}{c|}{2012} \\
\cline { 2 - 11 } & \multicolumn{4}{|c|}{ Quintiles de ingresos } & \multicolumn{5}{c|}{ Quintiles de ingresos } \\
\cline { 2 - 11 } & 1 & 2 & 3 & 4 & 5 & 1 & 2 & 3 & 4 & 5 \\
\hline Terciario & 2,61 & 6,31 & 11,08 & 14,58 & 19,49 & 3,94 & 8,4 & 13,28 & 13,67 & 16,77 \\
\hline Universitario & 1,45 & 2,72 & 5,39 & 12,03 & 30,93 & 1,66 & 3,69 & 9,93 & 11,46 & 26 \\
\hline
\end{tabular}

Fuente: elaboración propia con base en la Encuesta Permanente de Hogares del Instituto Nacional de Estadística y Censos, Argentina, 2006 y 2012, segundo trimestre. 
Efectivamente, los datos de la Encuesta Permanente de Hogares dan cuenta de que hay un avance significativo en el acceso de los jóvenes de bajos ingresos al nivel superior y un incremento en la obtención de la certificación educativa del nivel terciario, pero que todavía sigue pendiente la inclusión de los jóvenes en carreras universitarias. En este sentido, sería oportuno no solo sostener y aumentar la cobertura de becas para contribuir la permanencia en el sistema educativo de nivel superior, sino también la elaboración de dispositivos pedagógicos que acompañen las trayectorias educativas de estos jóvenes para apoyar la terminalidad de las carreras universitarias en distintos tramos.

\section{Amodo de cierre}

La mejora en la participación educativa de los sectores socioeconómicos más desfavorecidos constituye una de las metas relevantes en el esquema de la política educativa nacional; en este sentido, la tendencia al incremento de la escolarización es una clave auspiciosa de mejoría respecto a la ampliación de la cobertura. Tomando en cuenta las estadísticas oficiales de 2006 y 2012, los datos reflejan una ampliación de las posibilidades de acceso a la educación formal, así como un avance respecto a una mayor asistencia escolar de la población de jóvenes de menores ingresos. De acuerdo con el análisis realizado, desde mediados de la década de los noventa hasta 2012, las tendencias aprobarían el panorama de expansión de cobertura, pero a un ritmo menor de lo evidenciado hasta principios de la primera década de 2000, lo cual confirma cierto amesetamiento ${ }^{15}$.

En lo cuantitativo, la expansión educativa es un hecho todavía lejano, pues un porcentaje considerable de jóvenes no finaliza la escuela secundaria, aun cuando hoy es un ciclo obligatorio (no se ha llegado al 100 \% de la escolarización secundaria, y la meta de la universalización del nivel sigue pendiente). Pero este no es el único de los problemas que acecha el escenario educativo, pues en términos cualitativos, las grandes diferencias en relación con la calidad requieren atención. En este sentido, el incremento en el acceso al nivel superior enfrenta dificultades para la permanencia y la graduación. Los expertos sostienen que el abandono, sobre todo en los primeros años del nivel superior, está relacionado con la desarticulación entre los niveles (medio y superior) y, fundamentalmente, con la baja calidad de la enseñanza secundaria.

Respecto al nivel superior universitario y no universitario, existen brechas significativas entre los jóvenes que asisten al nivel superior de enseñanza y los que logran finalizar dicho nivel. Los porcentajes indican que si bien la representación de los sectores de menor nivel socioeconómico se ha ampliado, esta sigue siendo menor que para los grupos de mayores ingresos. Entretanto, los avances más significativos se dan en la evolución de los porcentajes relativos al acceso y no sobre la permanencia en el sistema educativo de este grupo

15 "La palabra amesetamiento indica, en términos económicos, que una variable estadística, hacia arriba o hacia abajo, se mantiene cercana a un promedio, entre crecimiento y caída" (Canale, 2012). 
social. Al observar los datos del periodo analizado, se destaca una tendencia al incremento de jóvenes incorporados a establecimientos terciarios, pero en lo referente a logros de culminación del nivel superior en la última década, no se verifican cambios sustantivos.

\section{Referencias}

Araujo, J. (2009). Articulación universidad-escuela secundaria como política pública: un análisis de los programas implementados por la Secretaría de Políticas. Revista Gestión Universitaria, 1(2). Recuperado el 10 de julio de 2016 de http://www.gestuniv.com.ar/gu_04/v2n1a2.htm

Canale, F. (28 de mayo de 2012). Informes del mal momento. ElLitoral.com. Recuperado de http:/www.ellitoral.com/index.php/diarios/2012/05/28/ economia1/ECON-02.html

Córica, A. y Otero, A. (2015). Perspectivas educativas y laborales de los jóvenes latinoamericanos. Revista Latinoamericana de Estudios Educativos, 45(2), 9-42.

Chiroleu, A. (2013). Usos y alcances de la democratización universitaria en Argentina y Brasil. En M. Unzué y S. Emiliozzi (comps.), Universidad y políticas públicas: ¿en busca del tiempo perdido? Argentina y Brasil en perspectiva comparada. Buenos Aires: Imago Mundi.

Dussel, I. (2006). Del amor y la pedagogía: notas sobre las dificultades de un vínculo. Buenos Aires: Del Estante Editorial.

Ezcurra, A. (2013). Igualdad en educación superior: un desafío mundial. Los polvorines: Universidad Nacional de General Sarmiento. Buenos Aires: Instituto de Estudios y Capacitación - Federación Nacional de Docentes Universitarios.

Finkel, S. (1977). La clase media como beneficiaria de la expansión del sistema educacional argentino 1880-1930. En G. Labarca et al., La educación burguesa. México, D. F.: Nueva Imagen.

García de Fanelli, A. y Jacinto, C. (2010). Equidad y educación superior en América Latina: el papel de las carreras terciarias y universitarias. Revista Iberoamericana de Educación Superior, 1(1), 58-75.

García de Fanelli, A. (2006). Acceso, abandono y graduación en la educación superior argentina, SITEAL (2005). Recuperado el 10 de julio de 2016 de www.siteal. iipe-oei.org/sites/default/files/siteal_debate_5_fanelli_artculo.pdf

Gessaghi, V. y Llinas, P. (2005). Democratizar el acceso a la educación superior. Buenos Aires: Centro de Implementación de Políticas Públicas para la Equidad y 
el Crecimiento. Recuperado el 18 de julio de 2016 de http://cippec.org/files/ documents/Otras\%20Publicaciones/democratizarelacceso_EDU.pdf

Kessler, G. (2014). Controversias sobre la desigualdad: Argentina, 2003-2013. Ciudad Autónoma de Buenos Aires: Fondo de Cultura Económica.

Krotsch, P. (2001). Educación superior y reformas comparadas. Buenos Aires: Universidad Nacional de Quilmes.

Mollis, M. (2008). La educación superior en Argentina: balance de un milenio. En La Educación Superior en el Mundo 3. GUNI. Madrid: Mundi-Prensa.

Peters, S. (2014). Educación, desigualdades y empleo: los límites de la expansión educativa. En F. Groisman y H. J. Burchardt, Desprotegidos y desiguales: ¿hacia una nueva fisonomía social? Ciudad Autónoma de Buenos Aires: Prometeo Libros.

Terigi, F. (2008): Los cambios en el formato de la escuela secundaria argentina: por qué son necesarios, por qué son tan difíciles. Revista Propuesta Educativa (29), 63-71.

Tenti Fanfani, E. (comp). (2008). Nuevos temas en la agenda de política educativa. Buenos Aires: Siglo XXI.

Tiramonti, G. (2004). Una nueva cartografía de sentidos para la escuela. En G. Tiramonti (comp.), La trama de la desigualdad educativa. Mutaciones recientes de la escuela media. Buenos Aires: Manantial. 


\section{$\mathbf{A}_{\text {nexos }}$}

Figura 1. Tasa neta de escolarización secundaria para Argentina por nivel de ingresos, 1998-2014

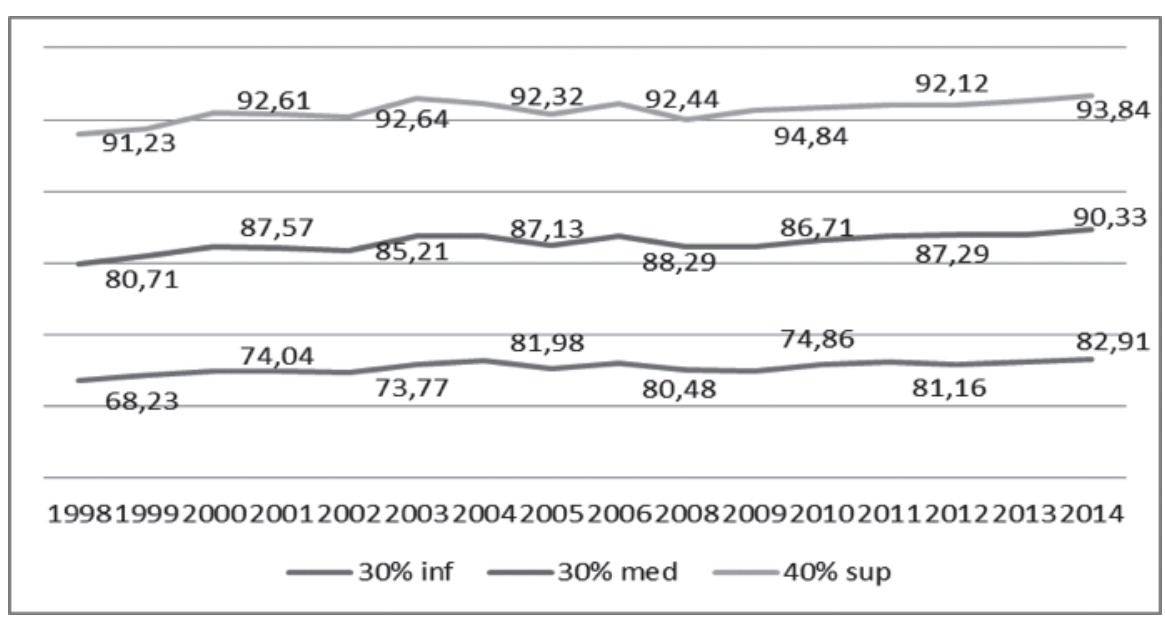

Fuente: Instituto Internacional de Planeamiento de la Educación - Organización de las Naciones Unidas para la Educación, la Ciencia y la Cultura; Organización de Estados Iberoamericanos. Con base en la Encuesta Permanente de Hogares del Instituto Nacional de Estadística y Censos, Argentina. 